\title{
LA GIMNASIA COMO CONTENIDO ESCOLAR: UN ANÁLISIS DE LOS PRIMEROS PROGRAMAS OFICIALES DE EDUCACIÓN FÍSICA EN URUGUAY (1911 Y 1923)¹
}

\author{
GYMNASTICS AS SCHOOL CONTENT: AN ANALYSIS OF THE FIRST OFFICIAL PHYSICAL \\ EDUCATION PROGRAMS IN URUGUAY (1911 AND 1923)
}

\section{A GINÁSTICA COMO CONTEÚDO ESCOLAR: UMA ANÁLISE DOS PRIMEIROS PROGRAMAS OFICIAIS DE EDUCAÇÃO FÍSICA NO URUGUAI (1911 E 1923)}

\section{Resumen}

\begin{abstract}
El trabajo presenta un análisis de los primeros programas oficiales de educación física escolar del Uruguay (1911 y 1923) en relación con la gimnasia. Nos interesa el recorrido programático de este contenido, asumiendo que dichos discursos estabilizados son portavoces de un contexto particular, abiertos a ser interpretados desde nuevas miradas. A través de estos documentos, que de una u otra manera marcan los lineamientos para la enseñanza, intentamos dar cuenta del recorrido histórico y conceptual de la gimnasia para comprender qué discursos la sustentan en su conformación curricular y qué recorte se realiza de ella como campo de saber.
\end{abstract}

Palabras clave: educación física; gimnasia; escolarización del cuerpo

\section{Abstract}

The purpose of this paper is to analyze the first official physical education programs in Uruguay (1911 and 1923) regarding gymnastics. Our goal is to perform a programmatic review of this content, assuming that these stabilized discourses are a reflection of a particular context that is open to interpretation from new perspectives. Through these documents, which set the guidelines for teaching, we try to give an account of the history and concept of gymnastics in order to understand the discourses supporting the structure of its curriculum, as well as its delimitation as a field of knowledge. It is argued that the implementation of gymnastics in Uruguayan schools followed the guidelines of European gymnastic movements, and that it was understood as a practical application of the knowledge of physiology, as well as channel of intervention in the population.

Keywords: physical education; gymnastics; schooling of the body

1 El presente artículo se desprende de las reflexiones trabajadas dentro de la línea Educación Física, Enseñanza y Escolarización del Cuerpo (Efeec). El proyecto a su vez forma parte de las investigaciones realizadas en el marco de la Red Internacional de Investigación Pedagógica en Educación Física Escolar (Reiipefe).

2 Estudiante del Doctorado en Educación de la Universidad Nacional de La Plata, Argentina. Profesora de Educación Física (Instituto Superior de Educación Física, Universidad de la República, Uruguay). Docente del Departamento de Educación Física y Prácticas Corporales (ISEF-UdelaR). Correo electrónico: viquialonso@gmail.com.

3 Estudiante de la Maestría en Educación Corporal de la Universidad Nacional de La Plata, Argentina. Profesor de Educación Física (Instituto Superior de Educación Física, Universidad de la República, Uruguay). Docente del Departamento de Educación Física y Prácticas Corporales (ISEF-UdelaR). Correo electrónico: pazinola@yahoo.com. 
O trabalho apresenta uma análise dos primeiros programas oficiais da educação física escolar do Uruguai (1911 e 1923) em relação com a ginástica. A pesquisa centra-se no percorrido programático deste conteúdo, assumindo que esses discursos estabilizados são porta-vozes de um contexto particular, com a possibilidade de ser interpretados desde novas perspectivas. Através destes documentos que estabelecem as diretrizes para o ensino, tentamos dar conta do percorrido histórico e conceitual da ginástica para compreender que os discursos a sustentam na sua conformação curricular e que é omitido desta como campo de sabe. Afirma-se que a implementação da ginástica na escola uruguaia seguiu as diretrizes dos movimentos ginásticos europeus, e que foi entendida como aplicação prática do sabes da fisiologia, assim como canal de intervenção na população.

Palavras chave: educação física; ginástica; escolarização do corpo

Fecha de recepción: 29 de julio de 2016

Fecha de aprobación: 27 de enero de 2017

Para citar este artículo:

Alonso, V. y Zinola, P. (2017). La gimnasia como contenido escolar: un análisis de los primeros programas oficiales de Uruguay (1911 y 1923). Lúdica Pedagógica, (25), 61-72. 


\section{INTRODUCCIÓN}

El siguiente trabajo intenta abordar uno de los aspectos que conforman el campo de la educación física escolar. Nos referimos a la dimensión curricular, analizada a partir de programas escolares oficiales. Nos interesa en particular el recorrido programático inicial que ha tenido la gimnasia como contenido de la educación física. En este artículo, presentamos el rastreo y análisis de la gimnasia en los programas de $1911^{4}$ y 1923 , documentos que se nos presentan como las dos primeras sistematizaciones que Uruguay realizó en la temática de la educación física escolar. A través de estos documentos, que de una u otra manera marcan los lineamientos para la enseñanza, sea en forma de manual escolar, plan de acción o proyecto de ejercicios físicos, intentamos comprender qué discursos sustentan la gimnasia escolar en sus inicios y qué recorte curricular se realiza de ella como campo de saber.

Partimos de concebir el currículo como "configuración discursiva específica de saber" (Bordoli y Blezio, 2007, p. 30), que de alguna manera se constituye en un universo lógicamente estabilizado donde todo tiene sentido, todo está representado y se presenta como una verdad. Pero el currículo también está abierto al acontecimiento, a la incesante reinterpretación por parte de los sujetos. Las modificaciones que presentan las distintas versiones programáticas son un insumo interesante para analizar las transformaciones de la propia disciplina pero también de la propia escuela en lo que esta toma, destaca, suprime. Tomar los programas oficiales como documentos de análisis responden a la necesidad de acercarnos al discurso estabilizado en cada etapa histórica de la educación física escolar, asumiendo la precariedad de las fuentes en la comprensión de un vasto contexto.

Es importante mencionar que no se trata de una investigación historiográfica, sino que las fuentes históricas manejadas servirán como contexto para el análisis de los discursos que de allí se desprenden acerca de

4 Existen antecedentes para este documento que se pueden rastrear en el programa escolar de Alejandro Lamas de 1897. Allí se presenta un breve apartado sobre "Ejercicios físicos" para el último ciclo escolar ( $7^{\circ}$ año) en el cual se prescriben los juegos libres y los ejercicios gimnásticos. Se menciona que estos últimos “... seguirán los principios y prácticas fundamentales del sistema sueco de gimnasia pedagógica" (Lamas, 1897, p. 60). Entendemos que esta breve reseña de la gimnasia escolar que aparece en el programa de 1897 opera como antecedente de la sistematización escrita en 1911 por el propio Lamas. la gimnasia como educación del cuerpo de una época particular. En este sentido, lo central es el saber y el conocimiento -la articulación teórica a partir de las fuentes- y no el relato histórico propiamente dicho.

\section{GIMNASIA Y ESCUELA 5}

Existen varios antecedentes de investigaciones sobre la educación del cuerpo en la escolarización de la infancia. En la región se destacan los trabajos de Ángela Aisenstein (2000) Ángela Aisenstein y (2006), Pablo Scharagrodsky $(2006,2007,2013)$ en Argentina, los de Valter Bracht (1996a, 1996b) y Carmen Soares (1994) en Brasil, y los de Raumar Rodríguez Giménez (2011, 2013) en Uruguay.

La escuela ha sido y es un lugar privilegiado de producción cultural, si bien no el único, y resulta clave la pregunta sobre los conocimientos en relación con el cuerpo que se ponen a circular en su interior.

Podemos decir en términos generales, estructurales (más allá del contexto particular) que la gimnasia se define como una práctica corporal sistemática, organizada, que reúne una serie de técnicas y ejercicios que buscan promover un trabajo equilibrado del cuerpo con un sentido de cuidado, desarrollo y enseñanza. La gimnasia en términos de proyecto moderno surgido en Europa desde finales del siglo XVIII ${ }^{6}$ se plantea como una forma de educar los cuerpos de los trabajadores de ciudades industriales en crecimiento, buscando prevenir enfermedades, vicios posturales, malos hábitos, mal uso del tiempo liberado del trabajo, entre otros factores principalmente de corte higienista y moral. Se trata de un conocimiento que luego, en el contexto del higienismo decimonónico dominante responderá a los últimos avances que en las ciencias realizaba principalmente la fisiología (Crisorio, 2007). Un rasgo característico es el de plantearse como una disciplina pensada para todos desde su conformación: como práctica homogeneizante para la educación de la ciudadanía, basada en la promesa de la salud nacional, o en expresión de Galak (2013), una empresa de profilaxis corporal

5 En Uruguay, el término escuela remite al periodo de enseñanza formal y obligatoria comprendido entre los 6 y los 12 años, también denominado enseñanza primaria.

6 La historia de la gimnasia es sumamente amplia y excede el propósito de este trabajo. De todas formas, si bien podemos rastrear este concepto desde la antigua Grecia (Mehl, 1986), el sentido antiguo y moderno de esta práctica varía sustancialmente. 
y sanidad moral. Gobierno del cuerpo junto al gobierno del pueblo son dos instancias de la gubernamentalidad -individuo y población-, y la gimnasia articula ambas desde principios del siglo XIX. Ante esta marcada necesidad de cientificismo de los conocimientos que se dejan entrar en la escuela, la gimnasia utilitaria, plausible de sistematicidad y mensurabilidad en cuanto a los esfuerzos impartidos, será quien esté más acorde a los intereses de la época ${ }^{7}$. Esta orientación de la gimnasia centrada en objetivos de carácter anátomo-fisiológicos y en mandatos morales, relacionados con la buena conducta de los ciudadanos, ingresará en nuestro país adquiriendo sentidos particulares a partir de necesidades locales. Los ejercicios físicos en la educación escolar serán comprendidos como el necesario complemento de una educación espiritual, siendo asimismo la base del desarrollo intelectual, moral y estético (Rodríguez Giménez, 2011).

Al hablar de gimnasia, estamos haciendo referencia a un contenido clave en la conformación de la identidad de un cuerpo de profesores, así como de la identidad del campo de la educación física en general (Alonso, 2012). No es casual que en el momento de indagar en la pregunta acerca de una posible matematización del campo, el saber por excelencia al cual remitir es precisamente la gimnasia (Torrón y Ruegger, 2012). Esto responde a una historia de legitimación y sistematización de prácticas y técnicas gimnásticas específicas que hasta el día de hoy continúan fuertemente asociadas a los profesores de educación física.

\section{LA GIMNASIA EN LOS PROGRAMAS ESCOLARES DEL URUGUAY: 1911 Y 1923}

En Uruguay, la gimnasia como disciplina específica que pone el conocimiento del cuerpo en un lugar central en la escuela cuenta con poco más de un siglo ${ }^{8}$. Se presenta como un contenido fundante de la educación física escolar, tal como aparece en los primeros documentos

7 En la diversidad de la gimnasia han habido y hay muchas manifestaciones que se alejan de este criterio cientificista, donde las alianzas se tejen más con expresiones cercanas al arte que a la ciencia. Sobre esta riqueza de fundamentos y prácticas, véase Langlade y Langlade (1986), Soares (2005), Madureira y Soares (2005).

8 En agosto de 1877 se sancionó el Decreto-Ley de Educación Común donde se incluyó un artículo sobre la gimnasia como materia para todos los grados. oficiales. Será, junto con los juegos, aquello que defina el espacio de la educación del cuerpo en la escuela desde finales del siglo XIX.

Hay acuerdos en asumir que la educación física ingresa al ámbito escolar uruguayo en 1905 a través de la figura de Alejandro Lamas, quien comenzó en ese año a dictar clases de gimnasia en las escuelas de Montevideo (Blanco, 1948). En 1910, Lamas presentó a la Dirección General de Instrucción Pública su "Proyecto de ejercicios físicos para las escuelas públicas". El mismo fue aprobado y puesto en práctica al año siguiente (Lamas, 1911), y se constituyó en el primer programa de educación física escolar a pesar de las dificultades en su implementación debidas a la falta de sistematicidad en la intervención, la ausencia de maestros especializados o de espacios disponibles.

A continuación, intentaremos dar cuenta del recorrido histórico y conceptual de la gimnasia como saber designado para ser enseñado, tal como aparece en los dos primeros programas escolares uruguayos. Entendemos que este recorte del saber que es un objeto a enseñar necesariamente implica una construcción, una serie de transformaciones para constituirse en un objeto de enseñanza. Según Chevallard (1998), todo proyecto social de enseñanza se forma dialécticamente con la identificación y designación de contenidos de saberes, como contenidos a enseñar. En este caso, focalizamos el análisis en aquello que se prescribe para la gimnasia, definido y estabilizado en los documentos programáticos que abordamos en este trabajo. Advertimos que en el momento de explicitar y definir dicho saber en relación con la gimnasia, los programas utilizan términos diversos, tal es el caso del concepto de ejercicio sistemado. Entendemos que son términos que han estado histórica y conceptualmente asociados a la gimnasia, lo que nos permite asumirlos como parte del campo y, por lo tanto, integrarlos al análisis.

En el cuadro 1 se resume lo escrito en cada uno de los programas de 1911 y 1923. Exponemos aquílos titulares que luego se despliegan en cada programa en numerosos ejercicios. 
Cuadro 1. Aspectos principales de los programas de 1911 y 1923.

\begin{tabular}{|l|l|}
\hline \multicolumn{2}{|c|}{1911} \\
\hline - ejercicios de orden: comprenden la & \\
buena distribución de los alumnos & \\
y organización disciplinaria, & \\
comprendiendo la táctica gimnástica & \\
y la táctica militar para los varones & \\
- ejercicios preparatorios: posiciones y & Ejercicios \\
actitudes fundamentales e iniciales & sistemados \\
- ejercicios fundamentales divididos & \\
en las siguientes series: ejercicios & \\
de miembros superiores, inferiores, & \\
combinados, de equilibrio, dorsales, & \\
abdominales, de lateralidad del & \\
tronco, de locomoción, de salto, de & \\
respiración. (Lamas, 1911, pp. 5-6) & \\
\hline & \multicolumn{1}{|c|}{ 1923* } \\
\hline - formaciones y despliegues gimnásticos & \\
- marchas y evoluciones \\
- carreras de conjunto formando \\
figuras y laberintos \\
- ejercicios calisténicos de \\
conjunto: por orden, rítmicos, \\
con música, donde sea posible. \\
- ejercicios calisténicos de conjunto con \\
manubrios, varas, clavas, aros, etc. \\
- ejercicio en aparatos pesados tales \\
como el caballo, el burro, la paralela, \\
las barras fijas, las anillas, etc. & Gimnasia \\
tales como el gimnasio al aire & \\
libre y la escalera horizontal." & \\
(Rodríguez, 1930, p. 28) & \\
\hline
\end{tabular}

${ }^{*}$ Para especificar los contenidos referidos a la gimnasia en este programa, incluimos también una edición posterior, publicada en 1930, que sin modificar el planteo original, desarrolla con mayor amplitud los contenidos presentados en la de 1923.

Fuente: elaboración propia a partir de los programas

\section{Una monotonía necesaria. Gimnasia en} el programa de 1911

El principal fundamento por el cual se incluye cualquier tipo de actividad corporal en la educación hacia finales del siglo XVIII es el beneficio fisiológico que el movimiento acarrea. Esto vale tanto para la gimnasia y sus "ejercicios sistemados" como para los "juegos metodizados", según expresiones utilizadas por Lamas en su programa de 1911. Los ejercicios sistemados se plantean complementarios a los juegos ${ }^{9}$.

9 El programa incluye: ejercicios sistemados, juegos metodizados dentro de la escuela, juegos metodizados fuera de la escuela y concursos gimnásticos periódicos.
La disputa entre lo artificial de la gimnasia y lo natural de los juegos queda planteada desde un comienzo. Se contrapone, a su vez, la actividad jugada y al aire libre a los ejercicios monótonos y analíticos de herencia sueca, si bien es esta la fuente de la cual se toman insumos ${ }^{10}$. Esta mirada tensa entre gimnasia y juegos queda explícita cuando Lamas expresa que

La cultura física que realizamos en las escuelas [...] es incompleta; el ejercicio sistemado no es positivamente útil, llena solo una parte de esa cultura, la que dice relación con el desarrollo armónico del cuerpo, la que cumple condiciones pedagógicas de orden, disciplina y desarrollo intelectual de ciertas facultades, la que llena condiciones higiénicas, estéticas y condiciones correctivas de posibles deformidades, pero no la otra, la que pide mucho oxígeno para la sangre, mucha actividad para los pulmones, mucha sudoración para la piel, mucho grito para las cuerdas vocales, viva excitación para los centros nerviosos y mucha, muchísima alegría para el espíritu y mucho, muchísimo regocijo de vida. Esta segunda parte sólo pueden darla los juegos y ejercicios al aire libre. (1912, pp. 42-43).

De todas formas, la gimnasia continúa ofreciendo algunas ventajas que le aseguran un lugar destacado en la escuela. Cuando se exaltan sus beneficios, se pone el acento en los aspectos de disciplinamiento del cuerpo y en la posibilidad de ejercitación que ofrece aun en espacios pequeños.

Los ejercicios físicos importan un descanso intelectual [...] favorecen de una manera positiva la disciplina, porque permiten la organización rápida de los alumnos por medio de los ejercicios de orden, les despierta el hábito del método, de la obediencia y de la regularidad, por el ritmo con que han de ejecutar todos los movimientos; además obligan al niño a procurarse actitudes correctas, a caminar con relativa desenvoltura y elegancia. [...] Finalmente suponen un medio artificial bueno, para reemplazar al natural de los juegos en la escuela en cuyos patios pequeños es imposible realizarlos. (Lamas, 1903, pp. 27-28).

10 El primer manual escolar que Lamas presentó en 1903 se basó en una traducción del francés del Manual de gimnasia sueca para uso de las escuelas primarias de Liedbeck (Gomensoro, 2009), lo que nos da una pista de la influencia de esta escuela gimnástica. 
En lo que compete entonces a los ejercicios gimnásticos, se proponen tres puntos: ejercicios de orden, preparatorios y fundamentales.

Los ejercicios de orden responden a la preocupación pedagógica por el disciplinamiento de los cuerpos. En esta preocupación que la gimnasia hace propia desde sus inicios, no importa tanto lo que se haga en términos de movimiento, sino que lo que se haga responda a una métrica determinada por una voz de mando. Importa educar los músculos para responder de forma rápida y eficaz a cualquier señal que se reciba. Podemos pensar incluso en una educación neuromuscular del anestesiamiento, en una acción estímulo-respuesta típica de la esperada en un ámbito militar, como el propio Lamas hace explícito al mencionar la "táctica militar para los varones". Esta forma de ejercitar los cuerpos, que llegará hasta nuestros días con la denominación de "formaciones", se basa justamente en la primacía de la forma sobre el contenido. Esto evidencia un interés estético que la gimnasia ha tenido desde sus inicios. Los movimientos que de ella se desprenden no se remiten solo a su carácter instrumental, sino que la forma que esos cuerpos adoptan es tanto o más relevante en ciertos contextos, que el propio efecto sobre el organismo. Esta idea se amolda perfectamente a la hipótesis de ritual formal que plantea Ljunggren (2011) como una de las pistas para comprender el éxito de la gimnasia de Ling en Suecia en el siglo XIX, a pesar de haber recibido fuertes críticas desde muy temprano. En palabras de Ljunggren:

Los rituales formales son estereotipados y altamente elaborados. Se distinguen, por ejemplo, por ciertos movimientos precisos, formas comunes y específicas de comportamiento y vestimenta, expresiones verbales y corporales. [...] refuerzan la idea de que algo existe más grande que el individuo [...] los sentimientos de poder surgen y los participantes se sienten fuertes. [...] Patrones rítmicos comunes crean un espíritu de equipo. (2011, p. 42).

Se hace presente en estas ejercitaciones la eficiente alianza que se tejió entre el discurso médico y el militar para dar fundamento a la gimnasia ${ }^{11}$. Podemos pensar

11 La relación de la gimnasia con el ámbito científico y militar es otro punto clave. Hay argumentos para cuestionar que haya sido la influencia militar la que condujo a Ling a procurar la sistematización racional de la gimnasia (Crisorio, 2010). Con mayor o menor éxito, las gimnasias tradicionales vinculadas a los grandes Estados-nación han hecho esfuerzos por desvincular sus que la búsqueda por un ordenamiento del cuerpo, tanto en términos epistémicos, estéticos como espaciales, ha sido una marca de la disciplina desde su conformación.

El segundo punto dentro de los ejercicios sistemados está comprendido por ejercicios preparatorios. El hecho de que la enseñanza de las posiciones iniciales requiera un punto especial en el programa nos indica la importancia que la gimnasia sueca adjudicó al control sobre el recorrido de los segmentos corporales en el espacio. Vigarello (2011) anuncia que "la verdadera novedad en el inicio del siglo XIX consiste en el análisis del movimiento: el cálculo de las fuerzas producidas así como el cálculo de las velocidades y de los tiempos" (p. 27). En este marco, este tipo de ejercicios "constituyen un programa interminable de aprendizajes secuenciados que establece una nueva disciplina en el universo pedagógico" (p. 28). Esta manera de desmenuzar el cuerpo, de analizarlo milimétricamente en la posición de cada segmento, solo puede concebirse en un contexto económico, social, cultural y político en el que el parámetro de comparación es la máquina, y toda la lógica de lo corporal queda atravesada por una inspiración mecánica del movimiento.

El tercer punto, ejercicios fundamentales, resalta la mirada que la gimnasia inaugura sobre el cuerpo, es decir, un cuerpo anatómicamente dividido en segmentos. Esto permitió la invención de movimientos parciales, de ejercicios localizados en una única articulación, de grupos musculares trabajados con independencia del resto, pero también al margen del gesto que se realiza para otra cosa, como podría ser el dominio de un objeto o la superación de un obstáculo. Lo que permite pensar, por ejemplo, hasta "1000 ejercicios diferentes para los brazos" (Laty [1996], citado en Vigarello, 2011, p. 29).

Un último aspecto que proponemos analizar aquí es el que refiere a la (des)atención puesta en la estimulación cardiovascular y respiratoria del organismo a través de los ejercicios gimnásticos. El hecho de que en este programa no aparezcan mencionadas ejercitaciones con ese fin responde a un claro posicionamiento de Lamas en su empresa de adaptar los sistemas sueco, francés y alemán, para constituir lo que casi se atreve a llamar "Sistema uruguayo de Gimnasia" (Lamas, 1912, p. 35). La propuesta de una acción fisiológica moderada responde

sistemas de este ámbito, ya que en muchos casos la gimnasia estuvo en manos de exmilitares. 
a una adecuación a nuestra población caracterizada por un "estado de pobreza orgánica de los niños uruguayos" (p. 30). Al respecto, este autor señala:

La gimnasia en la escuela no tiene, ni puede alcanzar y acaso no debe llegar a una acción fisiológica intensa. Su acción es eficaz, útil, modificatriz sólo en sentido relativo; podría serlo menos acercándose bastante a un resultado absoluto si variara fundamentalmente su procedimiento de aplicación o sea que los niños practicaran los ejercicios en traje adecuado, en locales amplios y propios, con tiempo bastante, bien alimentados antes y después de su venida y salida de la escuela. Pero, aún dentro de su relatividad es útil y necesaria, porque el resultado fisiológico es apreciable y su acción higiénica y pedagógica son evidentes ya que por ella se deriva el trabajo celular nervioso a la fibra muscular, se cambia el aire empobrecido del salón por el del patio y se consigue obtener el orden y la disciplina de las voluntades, indispensables para la buena marcha de la economía escolar. (1912, p. 38). [Énfasis en el original.]

Una vez más, la articulación entre higiene y disciplina será el pilar de la práctica de la gimnasia en el espacio escolar

Aire natural y científico. Gimnasia en el programa de 1923

En 1911 se creó la Comisión Nacional de Educación Física (CNEF), organismo clave en este recorrido histórico en nuestro país, ya que por muchos años se ocupó, entre otras cosas, de la promoción, gestión y programación de la educación física a nivel nacional abarcando ámbitos variados como las escuelas, las plazas de deportes o la formación de profesores de la disciplina. Este organismo (convertido a la órbita ministerial en junio de 2000), continuó rigiendo la educación física escolar hasta el año $2008^{12}$.

En 1923, en la misma línea que precedió a este programa, la CNEF presentó un plan de acción elaborado por su director técnico Julio J. Rodríguez. Este constituye un documento exhaustivo, en el cual intenta definir y

12 En el 2007 se realizó un pasaje a nivel organizativo y presupuestal de la estructura de la educación física comprendida en el Ministerio de Turismo y Deporte (antes, CNEF) al ámbito de la Administración Nacional de Educación Pública (ANEP). En el 2008 una comisión de este órgano elaboró un nuevo programa académico aún vigente. fundamentar científicamente la educación física. En el apartado "La educación física es una ciencia”, Rodríguez (1923, p. 9) escribe:

La educación física es una ciencia y sabemos que ésta no admite dogmas, ni principios absolutos, ni estrechez de escuelas. Ella está basada en las ciencias biológicas y psicológicas. Para aplicarla, primero se diagnostica y después se receta, y no como antes, que se aceptaban métodos y sistemas, como panaceas, que tanto se aplicaban a un niño en pleno crecimiento, como a un adulto que hacía vida sedentaria.

Como novedad, este documento propone un programa de intervención en distintos ámbitos, especialmente en las plazas de deporte ${ }^{13}$. Estos espacios al aire libre se consideran más adecuados para la educación física escolar ya que los edificios escolares "ni ahora ni nunca podrán llegar a reunir condiciones de higiene y de espacio al aire libre" (CNEF, 1913, en Scarlato, 2015, p. 88). Al mismo tiempo cumplen la función de "popularizar los ejercicios gimnásticos y hacerlos accesibles a la mayor parte de la población, que hoy no los practica" (CNEF [1911], citada en Scarlato 2015, p. 57). En síntesis, el fundamento de la realización de actividades en estos predios se basa en criterios preventivos (de enfermedades ocasionadas por el crecimiento demográfico) y de seguridad poblacional, al ofrecer espacios controlados para el juego infantil y la aglomeración de los adultos. Como expresa Scarlato (2015, p. 68), se trata de un "espaço de segurança para garantir o ótimo desenvolvimento do organismo, afastados da sujeira e imoralidade".

Este plan de acción se propuso como documento científico; esta intencionalidad se refleja en el sostén teórico proveniente de la fisiología, fundamentalmente, y de la psicología. Es importante detenernos en el discurso fisiológico propio de la época, ya que se edificó como la fuente principal de conocimiento de esta disciplina, portadora de un método natural de educación. La fisiología pregonaba por recuperar lo natural del cuerpo. Se debían suplantar los métodos calificados como

13 Las plazas de deportes, inauguradas en 1913 con el nombre de "Plazas vecinales de cultura física", constituyen espacios públicos que dan sustento al ejercicio del derecho a la actividad física, recreación y a la cultura deportiva. Constan en su mayoría de estructura deportiva al aire libre y algunas cuentan con gimnasios o piscinas cerradas. Las actividades están dirigidas a todo el público interesado y son organizadas por personal idóneo. En la actualidad hay 95 espacios con estas características en todo el país. 
artificiales por propuestas naturales, aptas para todos y de mayor eficacia (Crisorio, 2009). Este discurso fisiológico, en el cual la naturaleza opera como lugar de verdad y lo natural queda ligado a lo normal, es complementado por el discurso higienista en estrecho vínculo con lo pedagógico. "Sabemos -nos dice Rodríguez- que los principales fines de la gimnasia son los higiénicos, saludables, educacionales y correctivos" (1923, p. 34). Agrega además:

Cualquier factor o circunstancia que tienda a la supresión de los instintos y deseos naturales, o cualquier fracaso al tratar de satisfacerlos, impide el desarrollo normal o favorece el anormal. Durante la niñez, la escuela primaria y la plaza de deportes, deben tener bajo su responsabilidad el desarrollo y crecimiento normales de nuestra juventud. (p. 89).

Esta visión, donde lo natural se entiende como lo normal, debe interpretarse dentro de un contexto histórico particular. Con relación a este escenario, no es nuestra intención en este trabajo explicar los fenómenos histórico-políticos y culturales que afectaron a nuestra sociedad en aquella época. No obstante, es necesario atender y considerar, aunque sea superficialmente, la transformación social de principios de siglo Xx. Los grandes cambios sociales de la época generaron alteraciones en la vida cotidiana, en las costumbres y formas de vida. Cambios que se ajustaron a nuevos proyectos e intereses de un Estado que intentaba normalizar y domesticar la naciente sociedad de clases (Bouret y Remedi, 2009). Si nos referimos a nuevos proyectos e intereses, Barrán (1990), ubica como "los nuevos dioses" la higiene, la salud y la productividad. Al respecto, plantea: "trabajo, ahorro, disciplina. [...], salud e higiene del cuerpo fueron deificados a la vez que diabolizados el ocio, el lujo, el juego, la suciedad y la casi ingobernable sexualidad" (p. 34). Específicamente con relación al ámbito escolar, este autor señala que "la escuela vareliana [...] participó de esta convicción y fue uno de los agentes más eficaces en la internalización de este nuevo dios para los niños y las clases populares" (p. 35). Por lo tanto, la educación física, y en particular la gimnasia, justifica su presencia en la escuela en la medida en que responde a la necesidad social de enseñar la higiene, el mantenimiento de la salud y el fortalecimiento del cuerpo. Por lo tanto, teniendo presente este contexto, no es de extrañar que no encontremos a nivel conceptual grandes novedades en este nuevo programa. Estas primeras décadas de educación física en la escuela están claramente marcadas por las ideas positivistas de la época, junto con el auge del discurso médico higienista. La legitimidad de la intervención está dada por su efecto sobre el cuerpo de los niños, reequilibrando los malos hábitos posturales, posibilitando el normal desarrollo de los órganos y colaborando en la enseñanza de la higiene. Tal vez la diferencia esté en el alcance que este nuevo plan tiene, dada la diversidad de ámbitos a los que se destina: plazas de deporte, escuelas, universidad, patronato de delincuentes y menores.

Un punto que es particularmente resaltado, si bien continúa la línea de los planteos de Lamas, es lo que refiere a la relación con la naturaleza. El hecho de que la práctica sea pensada principalmente para las plazas de deporte marca el estrecho vínculo que se quiere establecer entre la actividad al aire libre, el tiempo librado del trabajo y una pedagogía del cuerpo. En lo que refiere a la gimnasia, como contenido del programa escolar, aparecen las formaciones, marchas, carreras o los ejercicios calisténicos ${ }^{14}$ con o sin aparatos. Podemos vincular la aparición de las marchas y carreras en este momento, tomando distancia del planteo de Lamas una década atrás, a la creciente mirada a la fisiología y la termodinámica como parámetros de intervención sobre el cuerpo. Se acrecienta la preocupación por el intercambio de oxígeno en pos de una mejor circulación en lo que algunos autores han marcado como el pasaje del cuerpo mecánico al cuerpo energético (cf. Gleyse, 2011).

La inclusión de la práctica médica en un cuerpo de ciencia físico-química despojó a la naturaleza del carácter matemático y mecánico que le habían atribuido Galileo y Descartes, y desplazó definitivamente el entendimiento del cuerpo humano como una máquina para concebirlo como un organismo en intercambio con el medio ambiente. (Crisorio, 2009, p. 45).

Se enfrentan así dos modelos que traerán como consecuencia que la gimnasia priorice o bien la forma del ejercicio o bien la activación orgánica. La primera se

\footnotetext{
14 Los ejercicios calisténicos se toman como sinónimos de gimnásticos. Este término fue acuñado por la tradición estadounidense a partir de sus interpretaciones de la gimnasia sueca y danesa. Su aparición en este programa evidencia la influencia que esta tradición ha tenido en nuestro medio, principalmente a través de la Asociación Cristiana de Jóvenes (cf. Giraldes, 2001, Dogliotti, 2011).
} 
corresponde con la metáfora de un cuerpo máquina muy utilizada hasta las primeras décadas del siglo $\mathrm{xx}$, y la segunda con la idea de un cuerpo energético que dejará las bases planteadas para el auge del movimiento deportivo a partir de la segunda mitad del siglo pasado.

Se vuelve a enfrentar aquí la tensión natural-artificial que acompaña a la gimnasia desde su surgimiento y la forma en que cada corriente reivindica los beneficios de una u otra expresión. Otra particularidad de este programa está marcada por la especial mención a los aparatos gimnásticos, sobre todo a los grandes aparatos al aire libre, de la mano de la creación de pórticos al estilo amorosiano ${ }^{15}$ que hasta la actualidad encontramos en el predio de las plazas de deportes. Esto nos plantea un giro en la práctica de la gimnasia en el que podemos suponer una mayor influencia francesa y alemana en cuanto a las destrezas logradas en estos grandes obstáculos, además de la tradicional influencia sueca. Es decir, la gimnasia comienza a desbordar los límites planteados por la tradición de Ling, donde lo central era la corrección postural en los movimientos geométricamente diseñados, y comienza a intervenir el espacio de formas distintas. Ya sea por la utilización privilegiada de los espacios abiertos en las plazas de deportes, por la incorporación de ejercicios volcados a la activación cardiovascular o por la realización de ejercicios de destreza en elementos como anillas, barras, cuerdas o trapecios típicos de los pórticos franceses.

\section{CONSIDERACIONES FINALES}

Este trabajo se focalizó en el análisis de la gimnasia escolar en los programas de 1911 y 1923. A partir de dicho análisis, podemos elaborar algunas reflexiones que nos permitan comprender las transformaciones ocurridas con esta particular forma de educación del cuerpo. Los discursos legitimadores que acompañaron a la gimnasia en las distintas épocas han sido clave para entender sus modificaciones a lo largo de la historia. En este sentido, encontramos que los programas estudiados, si bien con matices, responden a un mismo paradigma, en el cual el discurso fisiológico es determinante. El conocimiento de la fisiología impulsó una reforma científica en el campo de la educación física, modificando el concepto de naturaleza, que pasó a designar lo que debía considerarse

15 En referencia a los pórticos creados por Francisco Amorós y Ondeano (1770-1848), gran impulsor de la gimnasia francesa. normal respecto de los seres humanos (Crisorio, 2009). La gimnasia, entendida como aplicación práctica del saber de la fisiología, como canal de intervención en la población, no podía alejarse de estos supuestos. En este retorno a la naturaleza, lo natural se imagina como un estado original en el cual la "pureza" y la "robustez" de los pueblos nativos se destacan como ejemplos para la sociedad civilizada (Luz, 1997). Este imaginario se hace evidente en el programa de 1923 cuando se señala que "al estudiar la antropología puede encontrarse una completa evidencia para demostrar que el hombre primitivo, era infinitamente superior al hombre civilizado, en proporciones y estética del cuerpo [...]" (Rodríguez, 1923, p. 6).

La gimnasia entonces, en los programas de 1911 y 1923 , se legitima y opera fundamentalmente, junto con el discurso higiénico y moral, como un método para combatir los efectos de la civilización. La fisiología dará respuestas al qué, cómo, cuándo y dónde aplicar el método en pos de recuperar al buen salvaje. La justificación está dada con base en un beneficio fisiológico que el ejercicio controlado viene a ofrecer.

En especial, en el primer programa, se deja ver que por tedioso que pueda resultar este método de influencia sueca, no deja de ofrecer algunos beneficios que la escuela vareliana no desaprovecharía. Entre ellos se destacan la enseñanza del ordenamiento/ disciplinamiento del cuerpo en el espacio, la prevención y corrección de malas posturas y el descanso intelectual implicado en la compensación del trabajo en el aula.

La rigidez de las formas planteadas en el primer manual escolar, irá dando lugar a nuevas posibilidades de movimiento. Mientras que en 1911, el programa se centra únicamente en el ejercicio (su clasificación, descripción, momento, recorrido, segmento involucrado), en 1923 los ejercicios calisténicos se piensan sobre todo en función de la utilización o no de aparatos, ya sean portátiles o pesados. Esta relación distinta del cuerpo con el objeto, sumada a la inclusión de marchas y carreras, permite que el cuerpo de la gimnasia comience a rayar fuera de los límites geométricamente trazados por la tradición sueca de Ling.

Es interesante constatar que el modelo de la fisiología y la búsqueda de intervención sobre la naturaleza del cuerpo siguen siendo centrales en ambos documentos. Al mismo tiempo, el discurso fuertemente vinculado a la naturaleza como parámetro de normalidad irá dejando 
un terreno propicio para el crecimiento de la práctica deportiva en espacios públicos del país. Como ya se señaló, la implementación de la gimnasia en la escuela uruguaya siguió los lineamientos de los movimientos gimnásticos europeos. Tampoco fue ajena a las transformaciones que se produjeron principalmente por el auge del deporte desde mediados del siglo xx, lo que traería aparejado cierta pérdida de espacio de la gimnasia en la educación hacia la segunda mitad del mismo siglo.

\section{REFERENCIAS}

Aisenstein, A. (2000). Repensando la educación física escolar. Entre la educación integral y la competencia motriz. Buenos Aires: Novedades Educativas.

Aisenstein, A. y Scharagrodsky, P. (2006). Tras las huellas de la educación física escolar argentina. Buenos Aires: Prometeo.

Alonso, V. (2012). La cuestión de la gimnasia (o la gimnasia en cuestión): trayectos de una disciplina fuera y dentro del Instituto Superior de Educación Física. En Actas del XIV Encuentro Nacional IX Internacional de Investigadores en Educación Física. ISEF-UdelaR. Recuperado de http://www.isef.edu.uy/files/2014/10/ ALONSO-2.pdf.

Blanco, R. (1948). Educación física: un panorama de su historia. Montevideo: Impresora Adroher.

Barrán, J. (1990). Historia de la sensibilidad en el Uruguay (tomo 2). Montevideo: Ediciones de la Banda Oriental.

Bordoli, E. y Blezio, C. (Comps.). (2007). El borde lo (in) enseñable. Anotaciones para una teoría de la enseñanza. Montevideo: Psicolibros, Waslala.

Bouret, D. y Remedi, G. (2009). Escenas de la vida cotidiana. El nacimiento de la sociedad de masas (19101930). Montevideo: Ediciones de la Banda Oriental.

Bracht, V. (1996a). Educación física y aprendizaje social. Buenos Aires: Vélez Sárfield.

Bracht, V. (1996b). Educação física, no 1ํo Grau: conhecimento e especificidade. Revista paulista de educação física. Supl 2, p. 23-28. Recuperado de http://citrus. uspnet.usp.br/eef/uploads/arquivo/v10\%20supl2\%20 artigo4.pdf.
Chevallard, Y. (1998). La transposición didáctica. Del saber sabio al saber enseñado (3. ${ }^{a}$ ed.). Buenos Aires: Aique.

Crisorio, R. (2007). Educación física y biopolitica. Temas \& Matizes, 6(11) 67-78.

Crisorio, R. (2009). Educación física. En R. Crisorio y M. Giles (2009). Estudios críticos de educación física. La Plata: Ediciones Al margen.

Crisorio, R. (2010). Homero y Platón: dos paradigmas de la educación corporal (tesis de doctorado). Universidad Nacional de la Plata, Argentina.

Dogliotti, P. (2011). Cuerpo y currículum: discursividades en torno a la formación de docentes de educación física en Uruguay (1874-1948) (Tesis de maestría). Recuperado de http://posgrados.cse.edu.uy/sites/posgrados.cse. edu.uy/files/tesis_paola_dogliotti.pdf.

Galak, E. (2013). Herbert Spencer y la pedagogía integralista. Influencias en los inicios de la educación física argentina. En V. Varea y E. Galak (eds. Cuerpo y educación física. Perspectivas latinoamericanas para pensar la educación de los cuerpos. Buenos Aires: Biblos.

Giraldes, M. (2001). Gimnasia. El futuro anterior. De rechazos, retornos y renovaciones. Buenos Aires: Stadium.

Gleyse, J. (2011). La metáfora del cuerpo máquina en la educación física en Francia (1825-1935). En P. Scharagrodsky (comp.). La invención del "Homo gymnasticus": fragmentos históricos sobre la educación de los cuerpos en movimiento en occidente. Buenos Aires: Prometeo.

Gomensoro, A. (2009). Educación física escolar: aportes para su historia. En Educación Física...porque es un derecho. Montevideo: CEIP-ANEP.

Lamas, A. (1897). Programa de enseñanza primaria para escuelas urbanas. Montevideo: Dirección General de Instrucción Pública.

Lamas, A. (1903). Educación Física y manual de gimnasia escolar. Montevideo: Barreiro y Ramos.

Lamas, A. (1911). Proyecto de ejercicios físicos para las escuelas públicas. Montevideo: Barreiro y Ramos.

Lamas, A. (1912). Educación física é intelectual conexas. Un plan de educación física infantil. Montevideo: Barreiro y Ramos. 
Langlade, A. y Langlade, N. (1986). Teoría general de la gimnasia. Buenos Aires: Stadium.

Ljunggren, J. (2011). ¿Por qué la gimnasia de Ling? El desarrollo de la gimnasia sueca durante el siglo XIX. En P. Scharagrodsky (comp.). La invención del "Homo gymnasticus. Fragmentos históricos sobre la educación de los cuerpos en movimiento en Occidente. Buenos Aires: Prometeo.

Luz, M. (1997). Natural, racional, social. Razón médica y racionalidad científica moderna. Buenos Aires: Lugar Editorial.

Madureira, J. y Soares, C. (2005). Educação física, linguagem e arte: possibilidades de um diálogo poético do corpo. Movimento, 11(2), 75-88.

Mehl, E. (1986). Sobre la historia del concepto gimnástica. En: Citius, Altius y Fortius (separata del tomo IV, fascículo 2). Madrid: INEF Madrid.

Rodríguez, J. (1923). Plan de acción de la Comisión Nacional de Educación Física y conclusiones que se derivan del mismo. Montevideo: Imprenta Latina.

Rodríguez Giménez, R. (2011). Una conciencia y un corazón rectos en un cuerpo sano: educación del cuerpo, gimnástica y educación física en la escuela primaria uruguaya de la reforma. En P. Scharagrodsky (comp.). La invención del "Homo gymnasticus": fragmentos históricos sobre la educación de los cuerpos en movimiento en occidente. Buenos Aires: Prometeo.

Rodríguez Giménez, R. (2013). Educación del cuerpo y políticas educativas: de la formación superior al patio escolar. Revista Iberoamericana de Educación. El cuerpo en la escuela, 62, 107-118.
Scarlato, I. (2015). Corpo e tempo livre: as plazas vecinales de cultura física em Montevidéu (1911-1915) (tesis de maestria, inédita). Universidade Federal de Santa Catarina.

Scharagrodsky, P. (2007). El cuerpo en la escuela. Buenos Aires: Ministerio de Educación, Ciencia y Tecnología.

Scharagrodsky, P. (2011). La invención del "Homo gymnasticus": fragmentos históricos sobre la educación de los cuerpos en movimiento en occidente. Buenos Aires: Prometeo.

Scharagrodsky, P. (2013). Normalismo, "cultura física” y gimnasia en la Argentina. En G. Cachorro y E. Camblor. (Coord.). Educación física y ciencias. Abordajes desde la pluralidad. Buenos Aires: Biblos.

Soares, C. (1994). Educação física: raízes européias e Brasil. Campinas: Autores Associados.

Soares, C. (2005). Imagens da Educação no Corpo (3. ${ }^{\mathrm{a}}$ ed.). San Pablo: Autores Asociados.

Torrón, A. y Ruegger, C. (2012). La educación física como objeto matemizable. Didáskomai. Revista de Investigaciones sobre la Enseñanza, 3, 3.

Vigarello, G. (2011). La invención de la gimnasia en el siglo XIX: nuevos movimientos y nuevos cuerpos. En P. Scharagrodsky (comp.). La invención del "Homo gymnasticus": fragmentos históricos sobre la educación de los cuerpos en movimiento en occidente. Buenos Aires: Prometeo. 